\title{
One class of generalized boundary value problem for analytic functions
}

Pingrun Li*

\begin{tabular}{l}
\hline${ }^{*}$ Correspondence: \\
lipingrun@163.com \\
School of Mathematical Sciences, \\
Qufu Normal University, Jingxuanxi \\
Road 57, Qufu, Shandong 273165, \\
P.R. China
\end{tabular}

P.R. China

\begin{abstract}
In this paper, a boundary value problem for analytic functions with two unknown functions on two parallel straight lines is studied, the general solutions in the different domains as well as the conditions of solvability are obtained in class $\{1\}$, and the behaviors of solutions are discussed at $z=\infty$ and in the different domains, respectively. Therefore, the classic Riemann boundary value problem is extended further.
\end{abstract}

Keywords: boundary value problem for analytic functions; index; canonical function; the function class $\{1\}$

\section{Introduction and preliminaries}

Many mathematicians have studied the boundary value problems of analytic functions and formed a perfect theoretical system; see [1-7]. The boundary value problem of analytic functions on an infinite straight line has been studied in the literature, and there has been a brief description of boundary value problems of analytic function with an unknown function on several parallel lines. In this paper, we will put forward the boundary value problems of analytic functions with two unknown functions on two parallel lines and a general method different from the one in classical boundary value theory. Moreover, we will give and discuss the general solution and solvability conditions, which will generalize the classical theory of boundary value problems of analytic functions.

Let us describe the definitions of Plemelj formula and function class $\{1\}$ on an infinite straight line.

Definition 1.1 Assume that $\omega(x)$ is a continuous complex function on the real axis $X$. We say that $\omega(x) \in \hat{H}$ if the following conditions hold:

(1) For any sufficiently large positive number $M, \omega(x)$ satisfies $\omega(x) \in H$ on $[-M, M]$ (see [8] for the definition of $H$ ).

(2) $\left|\omega\left(x_{1}\right)-\omega\left(x_{2}\right)\right| \leq A\left|\frac{1}{x_{1}}-\frac{1}{x_{2}}\right|$, for any $\left|x_{j}\right|>M(j=1,2)$ and some positive real number $A$.

Under condition (2), we say that $\omega(x)$ satisfies the Hölder condition on $N_{\infty}$ and denote it $\omega(x) \in H\left(N_{\infty}\right)$, where $N_{\infty}=\{x:|x|>M\}$ is a neighborhood of $\infty$.

Definition 1.2 Assume that $\omega(x)$ is continuous on $(-\infty, \infty)$ and $\int_{-\infty}^{\infty}|\omega(x)| d x<+\infty$, then we say that $\omega(x) \in L_{1}(-\infty, \infty)$.

@ $2015 \mathrm{Li}$; licensee Springer. This is an Open Access article distributed under the terms of the Creative Commons Attribution License (http://creativecommons.org/licenses/by/4.0), which permits unrestricted use, distribution, and reproduction in any medium, provided the original work is properly credited. 
Definition 1.3 If $\omega(x)$ satisfies: (1) $\omega(x) \in \hat{H}$, (2) $\omega(x) \in L_{1}(-\infty, \infty)$, then we say that $\omega(x)$ belongs to the function class $\{1\}$.

Definition 1.4 Assume that $\omega(x) \in\{1\}$, then the integrals $\Omega^{+}(z)=\frac{1}{\sqrt{2 \pi}} \int_{0}^{+\infty} \omega(t) e^{i t z} d t$ and $\Omega^{-}(z)=\frac{1}{\sqrt{2 \pi}} \int_{-\infty}^{0} \omega(t) e^{i t z} d t$ are called the left and right one-sided Fourier integral, respectively.

Lemma 1.1 (see [1]) If $\omega(z) \in H$ with respect to any finite part of some infinite domain $D$, and $\omega(z)$ is analytic in any neighborhood of infinity, then $\omega(z) \in \hat{H}$.

Lemma 1.2 (see [2]) If $\omega(t)$ belongs to the class $\{1\}$, then the left and right one-sided Fourier integrals defined in Definition 1.4 are analytic when $\operatorname{Im} z>0$ and $\operatorname{Im} z<0$, respectively.

Lemma 1.3 (see [8]) If $\omega(x) \in \hat{H}$, we have the Cauchy type integral $\Omega(z)=\frac{1}{2 \pi i} \int_{-\infty}^{\infty} \frac{\omega(t)}{t-z} d t$, $z \notin(-\infty, \infty)$, then the following formula holds on the infinite straight line:

$$
\Omega^{ \pm}(x)= \pm \frac{1}{2} \omega(x)+\Omega(x), \quad \text { i.e., } \quad \Omega^{ \pm}(x)= \pm \frac{1}{2} \omega(x)+\frac{1}{2 \pi i} \int_{-\infty}^{\infty} \frac{\omega(t)}{t-x} d t .
$$

\section{Problem presentation}

Now, we put forward the boundary value problem of analytic functions on two parallel lines.

Without loss of generality, we assume that the two lines are parallel to the $X$-axis (otherwise, we can translate them into this case by a linear transformation), and denote them by $L_{1}, L_{2}$, where $L_{j}$ can be expressed by $\zeta=x+i l_{j}\left(x \in(-\infty, \infty), l_{2}<l_{1}\right.$ are real numbers) and take the direction from left to right as the positive direction. Let $L=L_{1}+L_{2}$.

We want to get functions $\Phi(z)$ and $\Psi(z)$ such that $\Phi(z)$ is analytic in $\left\{\operatorname{Im} z>l_{1}\right\} \cup\{\operatorname{Im} z<$ $\left.l_{2}\right\}, \Psi(z)$ is analytic in $\left\{z: l_{2}<\operatorname{Im} z<l_{1}\right\}$, and we have the following boundary value conditions:

$$
\begin{cases}\Phi^{+}(\zeta)=D_{1}(\zeta) \Psi^{-}(\zeta)+G_{1}(\zeta), & \text { when } \zeta \in L_{1}, \\ \Phi^{-}(\zeta)=D_{2}(\zeta) \Psi^{+}(\zeta)+G_{2}(\zeta), & \text { when } \zeta \in L_{2},\end{cases}
$$

where $L_{j}: \zeta=x+i l_{j}(j=1,2), x \in(-\infty, \infty)$.

Actually, (2.1) is a boundary value problem on two parallel straight $\operatorname{lines} \operatorname{Im} z=l_{1}, \operatorname{Im} z=$ $l_{2}$ with $\infty$ as a pole. Here $\Phi^{+}(\zeta)$ is the boundary value of analytic function $\Phi^{+}(z)$ which is analytic in $\left\{z: \operatorname{Im} z>l_{1}\right\}$ and belongs to the class $\{1\}$ on $L_{1}, \Phi^{-}(\zeta)$ is the boundary value of analytic function $\Phi^{-}(z)$ which is analytic in $\left\{z: \operatorname{Im} z<l_{2}\right\}$ and belongs to the class $\{1\}$ on $L_{2}$, and $\Psi^{ \pm}(\zeta)$ is the boundary value of analytic function $\Psi(z)$ which is analytic in $\left\{z: l_{2}<\operatorname{Im} z<l_{1}\right\}$ and belongs to the class $\{1\}$ on $L_{1}, L_{2}$, respectively. The functions $D_{1}(\zeta)$ and $D_{2}(\zeta)$ belong to $\hat{H}$ on $L_{1}, L_{2}$, respectively. The functions $G_{1}(\zeta)$ and $G_{2}(\zeta)$ belong to the class $\{1\}$ on $L_{1}, L_{2}$, respectively. Hence, for the functions appearing in (2.1) the one-sided limits exist when $x \rightarrow \infty$ on $L_{1}, L_{2}$.

It can be seen from (2.1) that the order of $\Phi(z)$ is equal to that of $\Psi(z)$ at infinity. Therefore, if the orders of $\Phi(z)$ and $\Psi(z)$ are $m$ at infinity, then such a problem can be denoted as $R_{m}$. Actually, problem $R_{0}$ and problem $R_{-1}$ are often discussed. On the problem $R_{0}$, both $\Phi(\infty)$ and $\Psi(\infty)$ are supposed to be finite and nonzero. On $R_{-1}$, both $\Phi(\infty)$ and $\Psi(\infty)$ are 
assumed to be zero. Such a problem $R$ is called regular if $D_{j}(\zeta)$ is not zero on $L$; otherwise, it is called irregular or of exception type.

Remark 2.1 Since the positive direction of $L_{j}$ is the direction from left to right, when the observer moves from left to right on $L_{j}$, the boundary values of left region of $L_{j}$ is positive boundary value, i.e., the positive boundary value of $\Phi(z)$ is the boundary value above $L_{1}$, and the negative boundary value of $\Phi(z)$ is ones below $L_{2}$. The positive or negative boundary values of $\Psi(z)$ can be defined in a similar way.

\section{Resolution}

We only consider problem $R_{0}$ in this paper. Hence, we assume $\Phi(\infty)$ and $\Psi(\infty)$ are finite and nonzero. For problem $R_{m}$, similar arguments can be used. In this paper, we only consider the normal case, that is, $D_{j}(\zeta)(j=1,2)$ does not have zeroes and poles on $L_{j}$. For the irregular case, similar discussions also work (see Section 2.5 of Chapter 2 in [1]). Equation (2.1) can be written as

$$
\left\{\begin{array}{l}
\Phi^{+}(\zeta)=D_{1}(\zeta) \Psi^{-}(\zeta)+G_{1}(\zeta), \quad \text { when } \zeta \in L_{1}, \\
\Psi^{+}(\zeta)=\frac{1}{D_{2}(\zeta)} \Phi^{-}(\zeta)-\frac{G_{2}(\zeta)}{D_{2}(\zeta)}, \quad \text { when } \zeta \in L_{2}
\end{array}\right.
$$

In order to unify, let $C_{1}(\zeta)=G_{1}(\zeta), C_{2}(\zeta)=G_{2}(\zeta) / D_{2}(\zeta)$, and the above equation can be transformed into

$$
\left\{\begin{array}{l}
\Phi^{+}(\zeta)=D_{1}(\zeta) \Psi^{-}(\zeta)+C_{1}(\zeta), \quad \text { when } \zeta \in L_{1} \\
\Psi^{+}(\zeta)=\frac{1}{D_{2}(\zeta)} \Phi^{-}(\zeta)-C_{2}(\zeta), \quad \text { when } \zeta \in L_{2}
\end{array}\right.
$$

By putting $\kappa_{1}=\operatorname{Ind}_{L_{1}} D_{1}(\zeta), \kappa_{2}=\operatorname{Ind}_{L_{2}} D_{2}(\zeta)$, and $\kappa=\sum_{j=1}^{2} \kappa_{j}$, we call $\kappa$ as the index of problem (2.1). Without loss of generality, we take three points $z_{0}, z_{1}, z_{2}$ on the $Z$ plane such that $l_{1}<\operatorname{Im} z_{1}, l_{2}<\operatorname{Im} z_{0}<l_{1}, \operatorname{Im} z_{2}<l_{2}$. Then we take the following piecewise function:

$$
Y_{1}(z)=\left\{\begin{array}{ll}
e^{\Omega_{1}(z)}, & \operatorname{Im} z>l_{1}, \\
\left(\frac{z-z_{0}}{z-z_{1}}\right)^{k_{1}} e^{\Omega_{1}(z)}, & \operatorname{Im} z<l_{1},
\end{array} \quad Y_{2}(z)= \begin{cases}\left(\frac{z-z_{0}}{z-z_{2}}\right)^{k_{2}} e^{\Omega_{2}(z)}, & \operatorname{Im} z>l_{2}, \\
e^{\Omega_{2}(z)}, & \operatorname{Im} z<l_{2},\end{cases}\right.
$$

here $\Omega_{j}(z)(j=1,2)$ is defined as follows:

$$
\Omega_{j}(z)=\frac{1}{\sqrt{2 \pi}} \int_{i l_{j}}^{+\infty+i l_{j}} r_{j}(t) e^{i t z} d t, \quad \text { when } l_{j}<\operatorname{Im} z_{j}
$$

and

$$
\Omega_{j}(z)=\frac{1}{\sqrt{2 \pi}} \int_{-\infty+i l_{j}}^{i l_{j}} r_{j}(t) e^{i t z} d t, \quad \text { when } \operatorname{Im} z_{j}<l_{j},
$$

where

$$
\begin{array}{ll}
r_{1}(t)=\frac{1}{\sqrt{2 \pi}} \int_{-\infty+i l_{1}}^{+\infty+i l_{1}} \log \tilde{D}_{1}(\tau) \cdot e^{-i \tau t} d \tau, & \tilde{D}_{1}(\tau)=\left(\frac{\tau-z_{0}}{\tau-z_{1}}\right)^{\kappa_{1}} D_{1}(\tau), \\
r_{2}(t)=\frac{1}{\sqrt{2 \pi}} \int_{-\infty+i l_{2}}^{+\infty+i l_{2}} \log \tilde{D}_{2}(\tau) \cdot e^{-i \tau t} d \tau, & \tilde{D}_{2}(\tau)=\left(\frac{\tau-z_{0}}{\tau-z_{2}}\right)^{\kappa_{2}} D_{2}^{-1}(\tau) .
\end{array}
$$


The function $\Omega_{j}(z)$ defined above is analytic on the complex plane except $L_{1}$ and $L_{2}$. The logarithmic function of the integrand has a certain analytic branch such that $\left.\log \frac{t-z_{0}}{t-z_{j}}\right|_{t=\infty}=0$; then $Y_{j}^{+}(z)$ and $Y_{j}^{-}(z)$ are analytic in $\left\{z: \operatorname{Im} z>l_{j}\right\}$ and $\left\{z: \operatorname{Im} z<l_{j}\right\}$, respectively. Moreover,

$$
\frac{Y_{1}^{+}(t)}{Y_{1}^{-}(t)}=\left(\frac{t-z_{0}}{t-z_{1}}\right)^{-k_{1}} e^{\Omega_{1}^{+}(t)-\Omega_{1}^{-}(t)}=\left(\frac{t-z_{0}}{t-z_{1}}\right)^{-k_{1}} \exp \left\{\frac{1}{\sqrt{2 \pi}} \int_{-\infty+i l_{1}}^{+\infty+i l_{1}} r_{1}(\zeta) e^{i t \zeta} d \zeta\right\}
$$

owing to

$$
V\left[r_{1}(t)\right]=\frac{1}{\sqrt{2 \pi}} \int_{-\infty+i l_{1}}^{+\infty+i l_{1}} r_{1}(\zeta) e^{i t \zeta} d \zeta
$$

by the representative of $r_{1}(t)$ as well as the relationship between Fourier transform and inverse Fourier transform, we have $V\left[r_{1}(t)\right]=\log \left[\left(\frac{t-z_{0}}{t-z_{1}}\right)^{k_{1}} D_{1}(t)\right]$, therefore

$$
\frac{Y_{1}^{+}(t)}{Y_{1}^{-}(t)}=\left(\frac{t-z_{0}}{t-z_{1}}\right)^{-k_{1}} \exp \left\{\log \left[\left(\frac{t-z_{0}}{t-z_{1}}\right)^{k_{1}} D_{1}(t)\right]\right\}=D_{1}(t)
$$

Similarly, one has

$$
\frac{Y_{2}^{+}(t)}{Y_{2}^{-}(t)}=D_{2}^{-1}(t)
$$

Putting (3.4), (3.5) into (3.2), we can obtain

$$
\begin{cases}\Phi^{+}(t)\left[Y_{1}^{+}(t)\right]^{-1}=\Psi^{-}(t)\left[Y_{1}^{-}(t)\right]^{-1}+C_{1}(t)\left[Y_{1}^{+}(t)\right]^{-1}, & t \in l_{1}, \\ \Psi^{+}(t)\left[Y_{2}^{+}(t)\right]^{-1}=\Phi^{-}(t)\left[Y_{2}^{-}(t)\right]^{-1}-C_{2}(t)\left[Y_{2}^{+}(t)\right]^{-1}, & t \in l_{2} .\end{cases}
$$

In (3.6), the first equality is multiplied by $\left[Y_{2}^{+}(t)\right]^{-1}$, the second one is multiplied by $\left[Y_{1}^{-}(t)\right]^{-1}$, then

$$
\left\{\begin{array}{l}
\Phi^{+}(t)\left[Y_{1}^{+}(t)\right]^{-1}\left[Y_{2}^{+}(t)\right]^{-1} \\
\quad=\Psi^{-}(t)\left[Y_{1}^{+}(t)\right]^{-1}\left[Y_{2}^{+}(t)\right]^{-1}+C_{1}(t)\left[Y_{1}^{+}(t)\right]^{-1}\left[Y_{2}^{+}(t)\right]^{-1}, \quad t \in l_{1}, \\
\Psi^{+}(t)\left[Y_{2}^{+}(t)\right]^{-1}\left[Y_{1}^{-}(t)\right]^{-1} \\
\quad=\Phi^{-}(t)\left[Y_{2}^{-}(t)\right]^{-1}\left[Y_{1}^{-}(t)\right]^{-1}-C_{2}(t)\left[Y_{2}^{+}(t)\right]^{-1}\left[Y_{1}^{-}(t)\right]^{-1}, \quad t \in l_{2},
\end{array}\right.
$$

denoting

$$
F_{1}^{+}(z)=\frac{1}{\sqrt{2 \pi}} \int_{i l_{1}}^{+\infty+i l_{1}} f_{1}(\tau) e^{i \tau z} d \tau, \quad F_{1}^{-}(z)=-\frac{1}{\sqrt{2 \pi}} \int_{-\infty+i l_{1}}^{i l_{1}} f_{1}(\tau) e^{i \tau z} d \tau
$$

where

$$
f_{1}(t)=\frac{1}{\sqrt{2 \pi}} \int_{-\infty+i l_{1}}^{+\infty+i l_{1}} \frac{C_{1}(\tau)}{Y_{1}^{+}(\tau) Y_{2}^{+}(\tau)} e^{-i \tau t} d \tau
$$


Using Lemma 1.2, we know that $F_{1}^{+}(z), F_{1}^{-}(z)$ are analytic in $\operatorname{Im} z>l_{1}, \operatorname{Im} z<l_{1}$, respectively. On $L_{1}$, we have

$$
F_{1}^{+}(t)-F_{1}^{-}(t)=\frac{C_{1}(t)}{Y_{1}^{+}(t) Y_{2}^{+}(t)} .
$$

Again we denote

$$
F_{2}^{+}(z)=\frac{1}{\sqrt{2 \pi}} \int_{i l_{2}}^{+\infty+i l_{2}} f_{2}(\tau) e^{i \tau z} d \tau, \quad F_{2}^{-}(z)=-\frac{1}{\sqrt{2 \pi}} \int_{-\infty+i l_{2}}^{i l_{2}} f_{2}(\tau) e^{i \tau z} d \tau
$$

where

$$
f_{2}(t)=\frac{1}{\sqrt{2 \pi}} \int_{-\infty+i l_{2}}^{+\infty+i l_{2}} \frac{C_{2}(\tau)}{Y_{1}^{-}(\tau) Y_{2}^{+}(\tau)} e^{-i \tau t} d \tau
$$

Similarly, $F_{2}^{+}(z), F_{2}^{-}(z)$ are analytic in $\operatorname{Im} z>l_{2}, \operatorname{Im} z<l_{2}$, respectively. On $L_{2}$, we obtain

$$
F_{2}^{+}(t)-F_{2}^{-}(t)=\frac{C_{2}(t)}{Y_{1}^{-}(t) Y_{2}^{+}(t)} .
$$

Then (3.7) may be reduced to

$$
\left\{\begin{array}{l}
\Phi^{+}(t)\left[Y_{1}^{+}(t) Y_{2}^{+}(t)\right]^{-1}-F_{1}^{+}(t) \\
\quad=\Psi^{-}(t)\left[Y_{1}^{-}(t) Y_{2}^{+}(t)\right]^{-1}-F_{1}^{-}(t), \quad t \in l_{1}, \\
\Psi^{+}(t)\left[Y_{1}^{-}(t) Y_{2}^{+}(t)\right]^{-1}+F_{2}^{-}(t) \\
\quad=\Phi^{-}(t)\left[Y_{1}^{-}(t) Y_{2}^{-}(t)\right]^{-1}+F_{2}^{-}(t), \quad t \in l_{2},
\end{array}\right.
$$

in the two sides of the first equation of (3.10), by adding $F_{2}^{+}(t)$; in the two sides of the second one by subtraction of $F_{1}^{-}(t)$, we have

$$
\left\{\begin{array}{l}
\Phi^{+}(t)\left[Y_{1}^{+}(t) Y_{2}^{+}(t)\right]^{-1}-F_{1}^{+}(t)+F_{2}^{+}(t) \\
\quad=\Psi^{-}(t)\left[Y_{1}^{-}(t) Y_{2}^{+}(t)\right]^{-1}-F_{1}^{-}(t)+F_{2}^{+}(t), \quad t \in l_{1}, \\
\Psi^{+}(t)\left[Y_{1}^{-}(t) Y_{2}^{+}(t)\right]^{-1}+F_{2}^{-}(t)-F_{1}^{-}(t) \\
\quad=\Phi^{-}(t)\left[Y_{1}^{-}(t) Y_{2}^{-}(t)\right]^{-1}+F_{2}^{-}(t)-F_{1}^{-}(t), \quad t \in l_{2},
\end{array}\right.
$$

the left side of the first equation of (3.11) is denoted by $M_{1}^{+}(t)$, the right side of one is denoted by $M_{1}^{-}(t)$; the left side of the second equation of (3.11) is denoted by $M_{2}^{+}(t)$, the right side of this one is denoted by $M_{2}^{-}(t)$. Let

$$
M_{1}(z)= \begin{cases}M_{1}^{+}(z), & \operatorname{Im} z>l_{1}, \\ M_{1}^{-}(z), & \operatorname{Im} z<l_{1},\end{cases}
$$

where

$$
\begin{aligned}
& M_{1}^{+}(z)=\Phi^{+}(z)\left[Y_{1}^{+}(z) Y_{2}^{+}(z)\right]^{-1}-F_{1}^{+}(z)+F_{2}^{+}(z), \\
& M_{1}^{-}(z)=\Psi^{-}(z)\left[Y_{1}^{-}(z) Y_{2}^{+}(z)\right]^{-1}-F_{1}^{-}(z)+F_{2}^{+}(z) .
\end{aligned}
$$


(1) We firstly consider the solutions of $M_{1}^{+}(z)$ and $M_{1}^{-}(z)$, respectively in $\operatorname{Im} z>l_{1}$ and $\operatorname{Im} z<l_{1}$.

Case: $k \geq 0$.

Since $\left[Y_{1}^{+}(z)\right]^{-1},\left[Y_{2}^{+}(z)\right]^{-1}$ are analytic in $\left\{z: \operatorname{Im} z>l_{1}\right\},\left[Y_{1}^{+}(z) Y_{2}^{+}(z)\right]^{-1}$ is analytic. It follows from $F_{j}^{+}(z)(j=1,2)$ is analytic that $M_{1}^{+}(z)$ is analytic. Hence, the $\lim _{z \rightarrow \infty}\left(\operatorname{Im} z>l_{1}\right) M_{1}^{+}(z)$ exists. Suppose that $M_{1}^{+}(z)=H_{1}(z)$, then

$$
\Phi^{+}(z)=Y_{1}^{+}(z) Y_{2}^{+}(z)\left[F_{1}^{+}(z)-F_{2}^{+}(z)+H_{1}(z)\right], \quad \operatorname{Im} z>l_{1},
$$

where $H_{1}(z)$ is analytic in $\left\{z: \operatorname{Im} z>l_{1}\right\}$ and the $\lim _{z \rightarrow \infty\left(\operatorname{Im} z>l_{1}\right)} H_{1}(z)$ exists.

When $\operatorname{Im} z<l_{1}$, since $\Psi^{-}(z)$ is defined in $\left\{z: l_{2}<\operatorname{Im} z<l_{1}\right\}, z_{0}$ is a $k$-order pole of $\left[Y_{1}^{-}(z) Y_{2}^{+}(z)\right]^{-1}(k>0)$. In order to ensure that $M_{1}^{-}(z)$ is bounded at a pole $z_{0}$, we can multiply by a factor $\left(z-z_{0}\right)^{k}$. Thus, it has a $k$-order at $z=\infty$, i.e., we have a polynomial with $k$ degree. Let $\left(z-z_{0}\right)^{k} M_{1}^{-}(z)=p_{k}(z)$, hence, $M_{1}^{-}(z)=\frac{p_{k}(z)}{\left(z-z_{0}\right)^{k}}$, i.e.,

$$
\Psi^{-}(z)\left[Y_{1}^{-}(z) Y_{2}^{+}(z)\right]^{-1}-F_{1}^{-}(z)+F_{2}^{+}(z)=\frac{p_{k}(z)}{\left(z-z_{0}\right)^{k}}, \quad \operatorname{Im} z<l_{1}
$$

where $p_{k}(z)=C_{0}+C_{1}\left(z-z_{0}\right)+\cdots+C_{k}\left(z-z_{0}\right)^{k}$ is a polynomial with degree no more than $\kappa$.

Case: $k<0$.

It follows from similar arguments as above that $M_{1}^{+}(z)$ is analytic in $\left\{z: \operatorname{Im} z>l_{1}\right\}$.

Because $\left[Y_{1}^{-}(z) Y_{2}^{+}(z)\right]^{-1}, F_{1}^{-}(z)$ and $F_{2}^{+}(z)$ are analytic in $\left\{z: \operatorname{Im} z<l_{1}\right\}$, so is $M_{1}^{-}(z)$. Moreover, $M_{1}^{+}(t)=M_{1}^{-}(t)$ on $L_{1}$. Hence, $M_{1}(z)$ is holomorphic in the whole complex plane and the $\lim _{z \rightarrow \infty} M_{1}(z)$ exists. By the Liouville theorem and the principle of analytic continuation, there is a constant $C$ such that $M_{1}(z)=C$, and one has

$$
\begin{array}{ll}
\Psi^{-}(z)=Y_{1}^{-}(z) Y_{2}^{+}(z)\left[F_{1}^{-}(z)-F_{2}^{+}(z)+C\right], & \operatorname{Im} z<l_{1}, \\
\Phi^{-}(z)=Y_{1}^{+}(z) Y_{2}^{+}(z)\left[F_{1}^{+}(z)-F_{2}^{+}(z)+C\right], & \operatorname{Im} z>l_{1} .
\end{array}
$$

Noticing that $z=z_{0}$ is a pole of $Y_{1}^{-}(z) Y_{2}^{+}(z)$ with order $-k, \Psi^{-}(z)$ has a singularity at $z_{0}$. In order to ensure that $\Psi^{-}(z)$ is analytic in $\left\{z: \operatorname{Im} z<l_{1}\right\}$ (in fact, for solving $\Psi^{-}(z)$ in the range that $\operatorname{Im} z<l_{1}$, we should only consider the case that $\left.l_{2}<\operatorname{Im} z<l_{1}\right)$. For the case that $k=-1$, it is sufficient to eliminate the singularity by putting $C=F_{2}^{+}\left(z_{0}\right)-F_{1}^{-}\left(z_{0}\right)$, then one can define $\Psi^{-}(z)$ in the following way:

$$
\Psi^{-}(z)=Y_{1}^{-}(z) Y_{2}^{+}(z)\left[F_{1}^{-}(z)-F_{2}^{+}(z)+F_{2}^{+}\left(z_{0}\right)-F_{1}^{-}\left(z_{0}\right)\right]
$$

When $\kappa \leq-2$, such a $C$ still cannot eliminate the singularity of $\Psi^{-}(z)$ at $z=z_{0}$. But the following condition should be satisfied:

$$
F_{2}^{+(q)}\left(z_{0}\right)-F_{1}^{-(q)}\left(z_{0}\right)=0, \quad q=1,2, \ldots,-\kappa-1,
$$

i.e.,

$$
\int_{-\infty+i l_{1}}^{i l_{1}} \tau^{q} f_{1}(\tau) e^{i \tau z_{0}} d \tau+\int_{i l_{2}}^{+\infty+i l_{2}} \tau^{q} f_{2}(\tau) e^{i \tau z_{0}} d \tau=0, \quad q=1,2, \ldots,-\kappa-1
$$


(3.15) is a solution of (2.1) if and only if (3.18) holds. Therefore,

$$
\begin{aligned}
& \text { as } k>0, \quad \Psi^{-}(z)=Y_{1}^{-}(z) Y_{2}^{+}(z)\left[F_{1}^{-}(z)-F_{2}^{+}(z)+\frac{p_{k}(z)}{\left(z-z_{0}\right)^{k}}\right], \quad \operatorname{Im} z<l_{1}, \\
& \Phi^{+}(z)=Y_{1}^{+}(z) Y_{2}^{+}(z)\left[F_{1}^{+}(z)-F_{2}^{+}(z)+H_{1}(z)\right], \quad \operatorname{Im} z>l_{1}, \\
& \text { as } k<0, \quad \Psi^{-}(z)=Y_{1}^{-}(z) Y_{2}^{+}(z)\left[F_{1}^{-}(z)-F_{2}^{+}(z)+C\right], \quad \operatorname{Im} z<l_{1}, \\
& \Phi^{+}(z)=Y_{1}^{+}(z) Y_{2}^{+}(z)\left[F_{1}^{+}(z)-F_{2}^{+}(z)+C\right], \quad \operatorname{Im} z>l_{1} .
\end{aligned}
$$

However, the solvability conditions should be satisfied (3.18) for $k<-1$. Similarly, we can define the following piecewise function:

$$
M_{2}(z)= \begin{cases}M_{2}^{+}(z), & \operatorname{Im} z>l_{2} \\ M_{2}^{-}(z), & \operatorname{Im} z<l_{2}\end{cases}
$$

where

$$
\begin{aligned}
& M_{2}^{+}(z)=\Psi^{+}(z)\left[Y_{1}^{-}(z) Y_{2}^{+}(z)\right]^{-1}-F_{1}^{-}(z)+F_{2}^{-}(z) \\
& M_{2}^{-}(z)=\Phi^{-}(z)\left[Y_{1}^{-}(z) Y_{2}^{-}(z)\right]^{-1}-F_{1}^{-}(z)+F_{2}^{-}(z) .
\end{aligned}
$$

(2) We secondly consider the solutions of $M_{2}^{+}(z)$ and $M_{2}^{-}(z)$, respectively, in $\operatorname{Im} z>l_{2}$ and $\operatorname{Im} z<l_{2}$.

Case: $k \geq 0$.

Since $Y_{1}^{-}(z)$ and $Y_{2}^{-}(z)$ are analytic in $\left\{z: \operatorname{Im} z<l_{2}\right\}$, so is $\left[Y_{1}^{-}(z) Y_{2}^{-}(z)\right]^{-1}$. It follows from $F_{j}^{-}(z)(j=1,2)$ being analytic that $M_{2}^{-}(z)$ is analytic. Hence, the $\lim _{z \rightarrow \infty}\left(\operatorname{Im} z<l_{2}\right) M_{2}^{-}(z)$ exists. Suppose that $M_{2}^{-}(z)=H_{2}(z)$, then

$$
\Phi^{-}(z)=Y_{1}^{-}(z) Y_{2}^{-}(z)\left[F_{1}^{-}(z)-F_{2}^{-}(z)+H_{2}(z)\right]
$$

where $H_{2}(z)$ is analytic in $\left\{z: \operatorname{Im} z<l_{2}\right\}$ and the $\lim _{z \rightarrow \infty}\left(\operatorname{Im} z<l_{2}\right) H_{2}(z)$ exists.

When $\operatorname{Im} z>l_{2}, z=z_{0}$ is a $k$-order pole of $\left[Y_{1}^{-}(z) Y_{2}^{+}(z)\right]^{-1}(k>0)$, then $z=z_{0}$ is a $k$-order pole of $M_{2}^{+}(z)$. By similar arguments to above, one has

$$
\Psi^{+}(z)\left[Y_{1}^{-}(z) Y_{2}^{+}(z)\right]^{-1}-F_{1}^{-}(z)+F_{2}^{+}(z)=\frac{p_{k}(z)}{\left(z-z_{0}\right)^{k}}
$$

Case: $k<0$.

Since $\left[Y_{1}^{-}(z) Y_{2}^{-}(z)\right]^{-1}$ has no singularity in $\left\{z: \operatorname{Im} z<l_{2}\right\}, M_{2}^{-}(z)$ is analytic in $\left\{z: \operatorname{Im} z<l_{2}\right\}$. Noticing that $\left[Y_{1}^{-}(z) Y_{2}^{+}(z)\right]^{-1}$ is analytic in $\left\{z: \operatorname{Im} z>l_{2}\right\}$, one finds that $M_{2}^{+}(z)$ is analytic in $\left\{z: \operatorname{Im} z>l_{2}\right\}$ and $M_{2}^{+}(t)=M_{2}^{-}(t)$ on $L_{2}$. Therefore, $M_{2}(z)$ is holomorphic in the whole complex plane and the $\lim _{z \rightarrow \infty} M_{2}(z)$ exists. By similar arguments to (1), there exists a constant $C$, such that $M_{2}(z)=C$ and

$$
\begin{array}{ll}
\Psi^{+}(z)=Y_{1}^{-}(z) Y_{2}^{+}(z)\left[F_{1}^{-}(z)-F_{2}^{+}(z)+C\right], & \operatorname{Im} z>l_{2}, \\
\Phi^{-}(z)=Y_{1}^{+}(z) Y_{2}^{+}(z)\left[F_{1}^{+}(z)-F_{2}^{+}(z)+C\right], & \operatorname{Im} z<l_{2} .
\end{array}
$$


Moreover, (3.18) is also a necessary condition for solvability. Hence,

$$
\begin{aligned}
& \text { as } k>0, \quad \Psi^{+}(z)=Y_{1}^{-}(z) Y_{2}^{+}(z)\left[F_{1}^{-}(z)-F_{2}^{+}(z)+\frac{p_{k}(z)}{\left(z-z_{0}\right)^{k}}\right] \\
& \Phi^{-}(z)=Y_{1}^{-}(z) Y_{2}^{-}(z)\left[F_{1}^{-}(z)-F_{2}^{-}(z)+H_{2}(z)\right], \\
& \text { as } k<0, \quad \Psi^{+}(z)=Y_{1}^{-}(z) Y_{2}^{+}(z)\left[F_{1}^{-}(z)-F_{2}^{+}(z)+C\right], \\
& \Phi^{-}(z)=Y_{1}^{+}(z) Y_{2}^{+}(z)\left[F_{1}^{+}(z)-F_{2}^{+}(z)+C\right] .
\end{aligned}
$$

Collecting results, for $k \geq 0$ and $l_{2}<\operatorname{Im} z<l_{1}$, one has

$$
\Psi(z)=Y_{1}^{-}(z) Y_{2}^{+}(z)\left[F_{1}^{-}(z)-F_{2}^{+}(z)+\frac{p_{k}(z)}{\left(z-z_{0}\right)^{k}}\right]
$$

and, for $k<0$ and $l_{2}<\operatorname{Im} z<l_{1}$,

$$
\Psi(z)=Y_{1}^{-}(z) Y_{2}^{+}(z)\left[F_{1}^{-}(z)-F_{2}^{+}(z)+C\right]
$$

where $p_{k}(z)$ and $C$ as above. For $z \in\left\{z: \operatorname{Im} z>l_{1}\right\}$,

$$
\Phi^{+}(z)=Y_{1}^{+}(z) Y_{2}^{+}(z)\left[F_{1}^{+}(z)-F_{2}^{+}(z)+H_{1}(z)\right]
$$

where $H_{1}(z)$ is analytic in $\left\{z: \operatorname{Im} z>l_{1}\right\}$ as $k \geq 0$ and the $\lim _{z \rightarrow \infty}\left(\operatorname{Im} z>l_{1}\right) H_{1}(z)$ exists. When $k<0, H_{1}(z) \equiv C$ (constant). For $z \in\left\{z: \operatorname{Im} z<l_{2}\right\}$,

$$
\Phi^{-}(z)=Y_{1}^{-}(z) Y_{2}^{-}(z)\left[F_{1}^{-}(z)-F_{2}^{-}(z)+H_{2}(z)\right]
$$

where $H_{2}(z)$ is analytic in $\left\{z: \operatorname{Im} z<l_{2}\right\}$ when $k \geq 0$ and $\lim _{z \rightarrow \infty}\left(\operatorname{Im} z<l_{2}\right) H_{2}(z)$ exists. $H_{2}(z) \equiv$ $C$ (constant) when $k<0$.

Hence we get the solution of the boundary value problem (2.1).

Theorem 3.1 The boundary value problem (3.1) with two unknown functions $\Psi(z)$ and $\Phi(z)$ on two parallel lines has a solution in $\left\{z: l_{2}<\operatorname{Im} z<l_{1}\right\}$ and $\left\{\operatorname{Im} z>l_{1}\right\} \cup\left\{\operatorname{Im} z<l_{2}\right\}$, respectively. Moreover, the general solution can be expressed by (3.33)-(3.36), where $Y_{j}^{ \pm}(z)$ $(j=1,2)$ is defined by (3.3) and $F_{j}(z)(j=1,2)$ are defined by (3.8) and (3.9). When $\kappa>-1$, $p_{k}(z)$ is a polynomial with $\kappa$ order, and when $\kappa \leq-1$, the necessary conditions for solvability still are (3.18). In all, the degree of freedom of the solution is $\kappa+1$.

\section{Further discussion on solution and solvability conditions}

In this section, we say more about the solution (3.33)-(3.36) of (2.1) and the solvability conditions.

(1) The case that the solution lies in $\operatorname{Im} z>l_{1}$ and $\operatorname{Im} z<l_{2}$.

As in (3.27) and (3.28), $\Phi^{+}(z)$ is analytic in $\left\{z: \operatorname{Im} z>l_{1}\right\}$ and $\Phi^{-}(z)$ is analytic in $\{z$ : $\left.\operatorname{Im} z<l_{2}\right\}$. No matter how we choose $\kappa$, the boundary value problem (2.1) is solvable and its solution can be expressed by (3.35)-(3.36).

(2) The case that the solution lies in $l_{2}<\operatorname{Im} z<l_{1}$. 
It can be seen from the expression of $\Psi(z)$ that $z_{0}$ is a $|\kappa|$-order pole of $Y_{1}^{-}(z) Y_{2}^{+}(z)$ when $\kappa<0$. In order to ensure (2.1) is solvable, one has $C=F_{2}^{+}\left(z_{0}\right)-F_{1}^{-}\left(z_{0}\right)$ when $k=-1$, i.e.,

$$
C=\frac{1}{\sqrt{2 \pi}} \int_{-\infty+i l_{1}}^{i l_{1}} f_{1}(\tau) e^{i \tau z_{0}} d \tau+\frac{1}{\sqrt{2 \pi}} \int_{i l_{2}}^{+\infty+i l_{2}} f_{2}(\tau) e^{i \tau z_{0}} d \tau
$$

When $k<-1$, the following $|\kappa|-1$ conditions are required:

$$
\int_{-\infty+i l_{1}}^{i l_{1}} \tau^{q} f_{1}(\tau) e^{i \tau z z_{0}} d \tau+\int_{i l_{2}}^{+\infty+i l_{2}} \tau^{q} f_{2}(\tau) e^{i \tau z z_{0}} d \tau=0, \quad q=1,2, \ldots,-\kappa-1
$$

Then $\Psi(z)$ is analytic in $\left\{z: l_{2}<\operatorname{Im} z<l_{1}\right\}$ and has a bounded solution. When $\kappa>0, z_{0}$ is a $\kappa$-order pole of $Y_{1}^{-}(z) Y_{2}^{+}(z)$, and therefore $Y_{1}^{-}(z) Y_{2}^{+}(z) \frac{p_{\kappa}(z)}{\left(z-z_{0}\right)^{\kappa}}$ is analytic in $\left\{z: l_{2}<\operatorname{Im} z<l_{1}\right\}$. Hence, $\Psi(z)$ is analytic in $\left\{z: l_{2}<\operatorname{Im} z<l_{1}\right\}$ and $\Psi(z)$ is a constant while $z=\infty$.

For $z \in\left\{z: l_{2}<\operatorname{Im} z<l_{1}\right\}, \Psi(z)$ can be defined by (3.33), (3.34) if $D_{1}(z)$ and $D_{2}^{-1}(z)$ are not zero. Otherwise, if $z_{1}^{*}, z_{2}^{*}, \ldots, z_{n}^{*}$ are common zero-points of $D_{1}(z)$ and $D_{2}^{-1}(z)$ with the orders $s_{1}, s_{2}, \ldots, s_{n}$, respectively, then $\Psi^{(j)}\left(z_{q}^{*}\right)=0\left(1 \leq q \leq n, 1 \leq j \leq s_{q}\right)$. Let $s=\sum_{q=1}^{n} s_{q}$. Then the following solvability conditions must be augmented.

As $\kappa \geq 0$, the following $\kappa+1$ element equations with unknown numbers $c_{0}, c_{1}, \ldots, c_{\kappa}$ :

$$
\left[\frac{p_{\kappa}(z)}{\left(z-z_{0}\right)^{\kappa}}\right]_{z=z_{q}^{*}}^{(j)}=\frac{j ! i^{j}}{\sqrt{2 \pi}}\left[\int_{-\infty+i l_{1}}^{i l_{1}} \tau^{j} f_{1}(\tau) e^{i \tau z} d \tau+\int_{i l_{2}}^{+\infty+i l_{2}} \tau^{j} f_{2}(\tau) e^{i \tau z} d \tau\right]
$$

As $\kappa<0$, the following condition is required:

$$
\int_{-\infty+i l_{1}}^{i l_{1}} \tau^{j} f_{1}(\tau) e^{i \tau z} d \tau+\int_{i l_{2}}^{+\infty+i l_{2}} \tau^{j} f_{2}(\tau) e^{i \tau z} d \tau=0
$$

$\left(j=0,1,2, \ldots, s_{q} ; q=1,2, \ldots, n\right)$, where $c_{0}, c_{1}, \ldots, c_{\kappa}$ are the coefficients of $p_{k}(z)$.

(3) The case of solutions at $z=\infty$.

In order to discuss the solution at $z=\infty$, we denote

$$
\begin{aligned}
& \gamma_{\infty}^{(1)}=\mu_{\infty}^{(1)}+i v_{\infty}^{(1)}=\frac{1}{2 \pi i}\left\{\log D_{1}\left(i l_{1}+\infty\right)-\log D_{1}\left(i l_{1}-\infty\right)\right\}, \\
& \gamma_{\infty}^{(2)}=\mu_{\infty}^{(2)}+i v_{\infty}^{(2)}=\frac{1}{2 \pi i}\left\{\log D_{2}\left(i l_{2}+\infty\right)-\log D_{2}\left(i l_{2}-\infty\right)\right\}, \\
& \mu_{\infty}=\mu_{\infty}^{(1)}+\mu_{\infty}^{(2)},
\end{aligned}
$$

where the $\operatorname{logarithm}$ function $\log D_{j}(\tau)$ takes some certain continuous branch when $\operatorname{Re} \zeta>$ 0 or $\operatorname{Re} \zeta<0$ such that $0 \leq \mu_{\infty}<1$.

If $z=\infty$ is a common node, it follows from $F_{j}(\infty)=0(j=1,2)$ that $F_{j}(\zeta)=\frac{F_{j}^{*}(\zeta)}{|\zeta|_{j}^{*_{j}^{*}}}, \mu_{j}^{*}<\mu_{\infty}^{(j)}$ and $F_{j}^{*}(\zeta) \in H$ near $z=\infty$. By the conditions of (2.1), one has $\Psi(\zeta) \in\{1\}$. Therefore, $\Psi(\infty)$ exists and is finite. Denote $F(\zeta)=F_{1}(\zeta)-F_{2}(\zeta)$. Note that $0 \leq \mu_{\infty}<1$. If $\mu_{\infty}>\frac{1}{2}$, it is clear that $Y_{1}^{-}(\zeta) Y_{2}^{+}(\zeta) F(\zeta)=O\left(1 /|\zeta|^{\mu_{\infty}-\varepsilon}\right.$ ) (where $\varepsilon$ is a positive number sufficiently small and $|\zeta|$ is large enough) and $Y_{1}^{-}(\zeta) Y_{2}^{+}(\zeta) \frac{p_{\kappa}(\zeta)}{\left(\zeta-z_{0}\right)^{\kappa}}=O(1)$. If $\mu_{\infty} \leq \frac{1}{2}$, in order to ensure that $\Psi(\zeta) \in\{1\}$, the coefficient $e_{k}$ of $p_{k}(z)$ should be taken as

$$
e_{k}=\frac{1}{\sqrt{2 \pi}} \int_{-\infty+i l_{1}}^{i l_{1}} f_{1}(\tau) e^{i \tau z} d \tau-\frac{1}{\sqrt{2 \pi}} \int_{i l_{2}}^{+\infty+i l_{2}} f_{2}(\tau) e^{i \tau z} d \tau, \quad \text { while } \kappa \geq 0 .
$$


For $\kappa<0$, the condition (4.2) should hold and $j=1,2, \ldots,|\kappa|$.

If $z=\infty$ is a special node, i.e., $\mu_{\infty}=0$, one can translate it into the case that $\mu_{\infty} \leq \frac{1}{2}$ as a common node. For the rest, similar arguments can be used [9].

As for the boundary value problem with $n$ unknown functions on $n(n>2)$ parallel lines, there is no essential difference for the solving method with the case $n=2$. We will not elaborate.

\section{Example}

In this section we consider one important example in practice. In (3.2), suppose

$$
\begin{aligned}
& D_{1}(\zeta)=D_{2}(\zeta)=1, \quad C_{1}(\zeta)=\frac{1}{1+\zeta^{2}}, \quad C_{2}(\zeta)=\frac{1}{2+\zeta^{2}}, \\
& L_{1}: \quad \zeta=0, \quad L_{2}: \quad \zeta=x+i \quad(-\infty<x<+\infty) .
\end{aligned}
$$

Without loss of generality, we assume that $z_{1}=\frac{3 i}{2}, z_{2}=\frac{-i}{2}, z_{0}=\frac{i}{2}$. Then we have $\kappa_{1}=\kappa_{2}=0$ and hence $\kappa=0$. Therefore, $\gamma_{j}(t)=0, \Omega_{j}(t)=0, Y_{j}(z)=1(j=1,2)$. In this case, by (3.8) and (3.9), we obtain

$$
\begin{aligned}
& f_{1}(t)=\frac{1}{\sqrt{2 \pi}} \int_{-\infty}^{+\infty} \frac{1}{1+\tau^{2}} e^{-i \tau t} d \tau=\frac{\sqrt{\pi} e^{-t}}{2} \\
& f_{2}(t)=\frac{1}{\sqrt{2 \pi}} \int_{-\infty+i}^{+\infty+i} \frac{1}{2+\tau^{2}} e^{-i \tau t} d \tau=\frac{\sqrt{\pi} e^{-\sqrt{2} t}}{2}
\end{aligned}
$$

and then

$$
\begin{aligned}
& F_{1}^{+}(z)=\frac{1}{\sqrt{2 \pi}} \int_{0}^{+\infty} f_{1}(\tau) e^{i \tau z} d \tau=\frac{i}{2 \sqrt{2}(z+i)}, \\
& F_{1}^{-}(z)=-\frac{1}{\sqrt{2 \pi}} \int_{-\infty}^{0} f_{1}(\tau) e^{i \tau z} d \tau=\frac{i}{2 \sqrt{2}(z-i)} .
\end{aligned}
$$

Similarly, we have

$$
F_{2}^{+}(z)=\frac{i}{2 \sqrt{2}(z+\sqrt{2} i)}, \quad F_{2}^{-}(z)=\frac{i}{2 \sqrt{2}(z-\sqrt{2} i)}
$$

Then we obtain the solutions of (3.2):

$$
\begin{aligned}
& \Psi(z)=\frac{i}{2 \sqrt{2}(z-i)}-\frac{i}{2 \sqrt{2}(z+\sqrt{2} i)}+C, \quad \text { when } 0<\operatorname{Im}<1, \\
& \Phi^{+}(z)=\frac{i}{2 \sqrt{2}(z+i)}-\frac{i}{2 \sqrt{2}(z+\sqrt{2} i)}+H_{1}(z), \quad \text { when } \operatorname{Im} z>1, \\
& \Phi^{-}(z)=\frac{i}{2 \sqrt{2}(z-i)}-\frac{i}{2 \sqrt{2}(z-\sqrt{2} i)}+H_{2}(z), \quad \text { when } \operatorname{Im} z<0,
\end{aligned}
$$

where $C$ is a constant, $H_{1}(z)=\left(1-\frac{\sqrt{2}}{2}\right) \frac{1}{1+z^{2}}, H_{2}(z)=0$. 


\section{Acknowledgements}

The author expresses sincere thanks to the referee(s) for the careful and details reading of the manuscript and very helpful suggestions, which improved the manuscript substantially.

Received: 5 August 2014 Accepted: 4 February 2015 Published online: 24 February 2015

\section{References}

1. Lu, JK: Boundary Value Problems for Analytic Functions. World Scientific, Singapore (2004)

2. Muskhelishvilli, NI: Singular Integral Equations. Nauka, Moscow (2002)

3. Lu, JK: On methods of solution for some kinds of singular integral equations with convolution. Chin. Ann. Math., Ser. B 8(1), 97-108 (1987)

4. Du, JY: On quadrature formulae for singular integrals of arbitrary order. Acta Math. Sci., Ser. B 24(1), 9-27 (2004)

5. Abreu-Blaya, R, Bory-Reyes, J, Brackx, F, De Schepper, H, Sommen, F: Boundary value problems for the quaternionic Hermitian in $\mathbb{R}^{4 n}$ analysis. Bound. Value Probl. (2012). doi:10.1186/1687-2770-2012-74

6. Lin, CC, Lin, YC: Boundary values of harmonic functions in spaces of Triebel-Lizorkin type. Integral Equ. Oper. Theory 79, 23-48 (2014)

7. Lu, JK: Some classes boundary value problems and singular integral equations with a transformation. Adv. Math. 23(5), 424-431 (1994)

8. Li, PR: On the method of solving two kinds of convolution singular integral equations with reflection. Ann. Differ. Equ. 29(2), 159-166 (2013)

9. Li, PR: The integral equations containing both cosecant and convolution kernel with periodicity. J. Syst. Sci. Math. Sci. 30(8), 1148-1155 (2010)

\section{Submit your manuscript to a SpringerOpen ${ }^{\circ}$ journal and benefit from:}

- Convenient online submission

- Rigorous peer review

- Immediate publication on acceptance

- Open access: articles freely available online

- High visibility within the field

Retaining the copyright to your article 